In questo spazio presentiamo alcuni fra i più recenti e autorevoli testi su tematiche incentrate sulle attività e sugli strumenti di marketing, pubblicati sia a livello nazionale che internazionale. Per eventuali segnalazioni contattare Gennaro Iasevoli via e-mail.

\section{Brondoni Silvio}

Marketing lexicon. Enciclopedia delle parole chiave di marketing

Clueb, 2011

"Marketing lexicon" è una raccolta ragionata di concetti di marketing, articolata secondo l'impostazione internazionale del marketing management. Il "Marketing lexicon" propone, con un lessico specialistico, l'interpretazione delle principali voci del marketing. Il testo è inoltre integrato da un dizionario delle parole chiave descritte nel libro e da una bibliografia specializzata di autori italiani e internazionali, per l'approfondimento dei diversi capitoli tematici che compongono il volume.

\section{Buratti Nicoletta, Ferrari Claudio}

La valorizzazione del patrimonio di prossimità tra fragilità e sviluppo locale. Un approccio multidisciplinare FrancoAngeli 2011

Cosa è patrimonio culturale di prossimità? A quali condizioni può innescare processi di sviluppo locale? Come costruire percorsi sostenibili di sviluppo endogeno a partire dalla valorizzazione del patrimonio di prossimità? Gli scritti contenuti nel volume sono organizzati in due parti: nella prima, vengono poste le basi concettuali e metodologiche per l'identificazione del patrimonio di prossimità come risorsa del territorio e la formulazione della strategia di valorizzazione in un'ottica di sostenibilità; nella seconda, si focalizza l'attenzione sul turismo culturale come strategia in grado di innescare processi virtuosi di sviluppo endogeno, analizzandone opportunità e limiti.

\section{Capano Giovanni}

Trade marketing e consumi fuori casa Ipsoa, 2011

Il focus del volume è sui canali distributivi dei consumi fuori casa, in particolare sulle best practice che l'Industria dovrebbe adottare per sviluppare campagne trade marketing oriented; in particolare, si approfondiscono sia la pianificazione, l'analisi e il controllo del trade marketing che il reale utilizzo delle leve del trade marketing mix. L'autore, analizzando la struttura della domanda (consumi e consumatori), e quella dell'offerta dei servizi legati ai consumi fuori casa (ovvero i luoghi di consumo e l'architettura dei diversi canali distributivi), evidenzia le differenze esistenti e le peculiarità tra i princi- 


\section{G. Iasevoli}

pali canali: Away From Home, Traditional Trade, Out of home, Indipendent Wholesalers, Ho.re.ca., ecc.

\section{Cioppi Marco}

PMI e nuove tecnologie della comunicazione. Applicazioni gestionali ed implicazioni strategiche

FrancoAngeli, 2011

Le ricerche condotte nel volume evidenziano che si è ancora molto lontani da un'adeguata valorizzazione nelle PMI delle potenzialità offerte dalle ICT, sebbene esista anche sotto questo profilo un'elevata eterogeneità. In particolare, le ICT vengono prevalentemente utilizzate solo per gestire in modo più efficiente alcune attività operative mentre rimane limitata la capacità di cogliere le possibilità di innovazione che esse offrono nella gestione dei processi decisionali ed operativi per la formulazione ed attuazione delle strategie, per acquisire nuovi clienti ed offrire migliori servizi, per accrescere il coordinamento interaziendale con i partner a monte ed a valle, per una maggiore efficacia delle politiche di comunicazione.

\section{Mattiacci Alberto, Militi Alessandro}

TV Brand. La rivoluzione del marketing televisivo

Lupetti, 2011

"Tv brand" è uno dei primi testi che approfondisce gli approcci alla base della costruzione di un brand televisi- vo e soprattutto aspira a rispondere alle tipiche domande di marketing: come si può differenziare in modo vincente un canale da un altro? Quali processi bisogna mettere in atto per trasformare un canale televisivo in TvBrand? In che modo può essere utilizzato per incrementare il fatturato dell'azienda? Chi sono gli attori di questa trasformazione e quali leve utilizzano?

\section{Valdani Enrico (a cura di)}

M@rketing Management. Progettare e generare valore per il cliente

Egea, 2011

Il volume, per la prima volta concepito per essere utilizzato in versione cartacea e multimediale, si propone come un punto di riferimento nella panoramica dei manuali di marketing management, perché unisce una singolare ampiezza di temi trattati a un approccio intuitivo ma rigoroso, che consente al lettore di cogliere sia la tradizionale anima qualitativa, sia la più attuale anima quantitativa che il marketing assume nella prassi delle aziende di successo. Il libro è organizzato in una sequenza di capitoli che ripercorrono le fasi del processo di analisi, formulazione e implementazione delle decisioni di marketing.

Ogni capitolo presenta un main case, un caso aziendale che viene utilizzato quale riferimento pragmatico per ricondurre, con un approccio induttivo e deduttivo, i principi teorici e paradigmatici. 


\section{MERCATI E COMPETITIVITÀ Rivista della Società Italiana Marketing MISSION DELLA RIVISTA}

Nata per merito della Società Italiana di Marketing questa Rivista vuole innanzitutto costituire una sede privilegiata della sua politica culturale e scientifica. Ciò tramite l'attivazione di tutte quelle sinergie e collaborazioni che possano garantirci di farne una rivista espressione del meglio di ciò che in Italia, e non solo, si produce in fatto di conoscenza e applicazioni del marketing. Ė ambizione della Rivista acquisire oltre che contributi delle migliori Scuole di marketing italiane anche un crescente numero di lavori di ricerca sviluppatisi in campo internazionale grazie a collaborazioni scientifiche ed editoriali con alcuni dei centri di eccellenza esistenti in Europa, negli Stati Uniti ed in prospettiva nel mondo asiatico.

II confronto tra diverse Scuole di marketing è assai opportuno in una fase in cui i paradigmi tradizionali e più consolidati del marketing management di origine anglosassone sono messi in discussione da nuovi modelli e prospettive di analisi e di gestione del mercato. Da tempo in Italia si sono sviluppate correnti di pensiero che si discostano dagli approcci più ortodossi e numerosi sono i contributi che, seppure ancora con una certa frammentazione, cercano di proporre e di sviluppare prospettive se non alternative almeno integrative e complementari a quelle più consolidate (il marketing relazionale, il marketing digitale, il co-operative marketing). In considerazione di tali aspetti la Rivista vuole essere un momento di aggregazione e di confronto sia tra studiosi sia tra questi e figure del mondo professionale e manageriale. Pur nascendo dall'interno di un'associazione scientifico-culturale la Rivista non è destinata a rimanere un campo di interessi e di contributi riservato. L'impegno è piuttosto quello di offrire per il suo tramite una visione aggiornata e scientificamente fondata dei campi e degli strumenti della scienza e delle tecniche del marketing, a beneficio di un più vasto e composito pubblico di autori e lettori. La "domanda di marketing" sta crescendo ovunque, nelle scuole e nelle università, nelle imprese e nel terzo settore del non profit, nella consulenza e nelle istituzioni pubbliche nazionali e locali. Non sempre a questa maggiore domanda corrisponde un'adeguata crescita dell'offerta di competenze e skills professionali, creando così disallineamenti e divari tra domanda e offerta. Non solo, ma spesso appare diffuso un "concetto di marketing" distorto e parziale nel quale vengono fatte convergere unicamente attività di comunicazione e di vendita, trascurandosi, per non dire ignorandosi, l'essenza e l'eterogeneità della cultura e delle attività di marketing management che investono una pluralità di processi di comprensione, generazione e trasmissione di ciò che è valore per il cliente. Anche in considerazione di ciò la Rivista vuole rispondere offrendo contributi preziosi per l'elevazione e l'aggiornamento culturale e scientifico di coloro che intendono occuparsi di marketing in modo specialistico e professionale, nella scuola e nell'università così come nel mondo produttivo e istituzionale.

\section{NORME REDAZIONALI}

Per una maggiore chiarezza informativa si precisa che:

- la rivista accoglie solo articoli originali;

- gli editoriali "ad invito" vengono pubblicati su invito della Redazione della Rivista;

- i saggi per le sezioni tematiche sono sottoposti alla valutazione di due referees anonimi ai fini dell'accettazione per la pubblicazione; in caso di discordanza delle due valutazioni è previsto il ricorso ad un terzo referee; 
- i saggi che rientrano tra "gli altri contributi" al di fuori delle sezioni tematiche sono sottoposti alla valutazione di due referees anonimi ai fini dell'accettazione per la pubblicazione;

- si invitano gli autori a tenere presente con attenzione il dibattito eventualmente già sviluppato in precedenza dalla Rivista nella tematica oggetto del paper candidato per la pubblicazione.

Gli articoli proposti per la pubblicazione devono conformarsi alle seguenti norme redazionali

1) Gli articoli devono essere inviati alla rivista via e-mail a entrambi gli indirizzi: roberta.sebastiani@unicatt.it e annalisa.tunisini@uniurb.it e al paper deve essere allegata un'autocertificazione sull'originalità del lavoro e sul rispetto delle norme redazionali sotto indicate (via mail, in formato pdf con firma originale, o copia originale per posta ordinaria)

2) Nome, afferenza istituzionale, indirizzo e recapiti telefonici dell'autore (o degli autori) ed eventuali annotazioni riguardanti l'articolo devono essere chiaramente indicati e devono essere inseriti in nota a piè di prima pagina (per queste note si utilizzano i simboli ${ }^{*} e^{* *}$, mentre per le note nel testo si utilizzano i numeri progressivi ricominciando da 1 a ogni articolo).

3) Degli articoli deve essere fornito un abstract in italiano e un abstract in inglese (con titolo in inglese) di circa 800 battute l'uno (si ricordi che per "battute" si intendono anche gli spazi). Ogni riassunto deve contenere in modo chiaro i punti salienti dell'articolo, e deve essere espresso col soggetto in terza persona (esempi: "L'autore sostiene che"). All'inizio dell'Abstract in inglese deve comparire il titolo dell'articolo tradotto in inglese.

4) Alla fine del riassunto e dell'abstract si devono scrivere sei "parole chiave" e sei "key words", rispettivamente, che indichino con chiarezza gli argomenti trattati (queste parole chiave servono per la indicizzazione dell'articolo nelle banche dati internazionali, e vengono anche utilizzate per gli indici analitici dell'annata).

5) I singoli saggi comprensivi di note e riferimenti bibliografici non devono superare le $\mathbf{5 0 . 0 0 0}$ battute (spazi inclusi).

L'inserimento di eventuali tabelle/grafici/figure non deve superare le 3 cartelle di lunghezza.

6) Impostazione testo e citazioni. Sono previsti 3 tipi di carattere: normale, corsivo, grassetto (detto anche neretto). Il corsivo va usato per le parole in lingua straniera di uso non comune e anche per evidenziare parole o frasi brevi dando loro una particolare enfasi. Per dare maggiore risalto a parole o frasi, e anche per citazioni non letterali, è preferibile usare le virgolette inglesi ("virgolette inglesi"), mentre le citazioni esatte vanno tra virgolette caporali («virgolette caporali»).

\section{7) Caratteri del testo}

Corpo del testo: Times New Roman, corpo 10, giustificato, interlinea singola, attivando il controllo delle righe isolate

Titolo del saggio: Times, corpo 12, corsivo, centrato

Titoli paragrafi: Times, corpo 10, neretto, all. a sinistra, 2 interlinee bianche sopra e 1 sotto

Sottoparagrafi: Times, corsivo, corpo testo, all. a sx., 2 int. bianche sopra e 1 sotto 
Numero pagina: Times, corpo testo, corsivo, centrato, con nessun rientro, senza cornice

Note (a piè di pagina): Times, corpo 9, interlinea singola, numerate progressivamente dalla nota $\mathrm{n} .1$

Riferimenti bibliografici: times, corpo 10, interlinea singola, prima riga sporgente $0,5 \mathrm{~cm}$

Intestazione e piè di pagina: times corpo 9

8) I riferimenti bibliografici inseriti direttamente nel testo verranno riportati col cognome dell'autore seguito da uno spazio e dall'anno della prima pubblicazione in lingua originale tra parentesi tonde - "Freud (1899) disse che..." - oppure col cognome dell'autore tra parentesi seguito da una virgola, spazio e anno (Freud, 1899). Se si vuole indicare la pagina, questa va indicata in parentesi nel testo preceduta da "p." dopo l'anno seguito da una virgola, e non nella bibliografia alla fine dell'articolo. Se gli autori sono due, devono essere scritti entrambi e collegati da "e", che può essere usata anche per collegare gli ultimi due autori se sono tre. Se gli autori sono più di tre, si scrive il cognome del primo autore seguito da et al. (in corsivo dal latino et alii).

9) I Riferimenti bibliografici a fine testo devono essere elencati senza numerazione alla fine del testo in ordine alfabetico secondo il cognome dell'autore e, per ciascun autore, nell'ordine cronologico di pubblicazione delle opere (per opere dello stesso autore pubblicate nello stesso anno, si usino le indicazioni a, b, c). I riferimenti bibliografici vanno redatti secondo le regole desumibili dai seguenti esempi:

\section{Volume:}

Beck A.T., Rush A.J., Shaw B.F. and Emery G. (1979). Cognitive Therapy of Depression. New York: Guilford Press (trad. it.: Terapia cognitiva della depressione. Torino: Boringhieri, 1987).

\section{Curatela, un autore:}

Merini A., a cura di (1977). Psichiatria nel territorio. Milano: Feltrinelli.

Curatela, più autori:

Boltanski L., Claverie E., Offenstadt N. and Van Damme S., a cura di (2007). Affaires, scandales et grandes causes. De Socrate à Pinochet. Paris: Stock.

\section{Saggio da curatela:}

Liotti G. (1985). Un modello cognitivo-comportamentale dell'agorafobia. In: Guidano V.F. e Reda M.A., a cura di, Cognitivismo e psicoterapia. Milano: FrancoAngeli.

\section{Saggio da rivista:}

Minguzzi G.F. (1986). È possibile valutare i risultati della psicoterapia? Giornale Italiano di Psicologia, 13, 1: 7-13.

Testo non pubblicato:

Benedetti G. (1988). "Intervento nel dibattito sulla relazione di John Gunderson al Convegno Internazionale New Trends in Schizophrenia", Bologna, 14-17 aprile (incisione su nastro).

\section{Volume o articolo da sito Internet:}

Si seguono le stesse indicazioni come nel caso di volumi e articoli stampati, con l'aggiunta di: testo disponibile al sito: http://www...(ultimoaccesso/gg/mm/aaaa)

10) Figure, tabelle e grafici: devono essere inseriti man mano nel testo, in un formato che consenta successivi eventuali interventi.

Le didascalie devono essere impostate in times, corpo 10 corsivo, allineato a sinistra.

La tabella sarà impostata in 8 o 9, times, testatina in alto: corsivo, con filetto nero sopra e sotto (vedi esempio). 
ESEMPIO DI TABELLA:

Tab. 3 - Distribuzione percentuale ....

\begin{tabular}{lccc}
\hline & Maschi & Femmine & Totale \\
\hline Abcde & 48,1 & 44,1 & 46,1 \\
Fghi & 44,7 & 44,3 & 44,5 \\
Lmno & 7,2 & 11,6 & 9,4 \\
Totale & 100 & 100 & 100 \\
$\mathrm{~N}$ & 1.153 & 1.208 & 2.361 \\
\hline
\end{tabular}

11) L'impostazione pagina I testi in forma definitiva devono essere inviati alla Direzione della Rivista in formato digitale in WORD (non è valido il formato PDF) via e-mail.

L'impostazione della pagina dovrà essere la seguente:

- $\quad$ margine superiore $\mathrm{cm} \mathrm{5}$; inferiore $\mathrm{cm} \mathrm{6,2;} \mathrm{sinistro} \mathrm{cm} \mathrm{4,4;} \mathrm{destro} \mathrm{cm} \mathrm{4.4}$.

- intestazione: $\mathrm{cm} 0$

- piè di pagina: $\mathrm{cm} \mathrm{5,5}$

- rilegatura: 0

- carattere 10 per il testo e 9 per le note; il tutto in Times new roman

- interlinea: singola

II testo risulterà di cm 12,2 di larghezza e 18,5 d'altezza. II numero di pagina sarà centrato e posizionato $\mathrm{a} \mathrm{cm} 0,8$ dal piede dell'ultima riga.

Ringraziamo per la collaborazione: seguire tali indicazioni consente alla redazione di risparmiare sui tempi di pubblicazione (e sui costi relativi).

Redattore Capo: Roberta Sebastiani - Università Cattolica del Sacro Cuore Segreteria di Redazione: Raffaella Bellani Società Italiana di Marketing c/o Facoltà di Economia - Area Marketing Via J.F. Kennedy 6, 43100 Parma Mob. 3464010937 Tel. 0521032399 fax 0521032302 e-mail: info@simktg.it 